\title{
DE L'HYPERTEXTE AU DESIGN MONOPAGE: UNE TRANSITION SÉMIOTIQUE
}

\section{FROM HYPERTEXT TOWARDS SINGLE-PAGE DESIGN: A SEMIOTIC TRANSITION}

VIVIEN LLOVERIA*

RÉSUMÉ: Dans la communauté des concepteurs de site web (webdesigners), le design de site dit monopage (single-page design, one-page design ou pageless design) apparaît comme la révolution de ces dernières années, au point de prétendre détrôner le genre numérique de l'hypertexte. Dans la mesure où elle expose une transition entre tradition hypertextuelle et nouveauté du design monopage, cette période offre l'opportunité d'étudier la relation entre l'émergence d'un dispositif techno-sémiotique et les systèmes de valeurs mobilisés jusque-là par les concepteurs et les lecteurs de sites web. Nous chercherons notamment à démontrer comment l'abandon de l'hypertexte, au profit du design monopage, reflète un souci de concevoir l'hypertexte non plus comme le monde interconnecté décrit par les scientifiques, mais comme une expérience de lecture/écriture singulière commentée par les usagers.

\footnotetext{
* Enseignant-chercheur au CeReS - Centre de Recherches Sémiotiques de Limoges. E-mail: vivien.lloveria@unilim.fr.
} 
MOTS-CLÉS: Sémiotique. Hypertexte. Design Monopage. Webdesign.

ABSTRACT: In the web designers' community, the single-page design (one-page design or pageless design) seems to be the revolution of the last years, professing the disappearance of hypertext. As a transition between the tradition of hypertext and the novelty of single-page design, this period gives us the opportunity to study the relationship between the emergence of a techno-semiotic device and the value systems associated to readers and web designers. Our aim is to show how the abandon of hypertext in favour of single-page design reflects a change of thinking the hypertext, no longer seen as an interconnected world described by scientists, but as a single reading/writing experience commented by users.

KEYWORDS: Semiotics. Hypertext. One page - Pageless Single-page design. Webdesign.

\section{Introduction}

Dans la communauté des concepteurs de site web (webdesigners), le design de site dit monopage (single-page design, one-page design ou pageless design) apparaît comme la révolution de ces dernières années, au point de prétendre détrôner le genre numérique de l'hypertexte. Dans la mesure où elle expose une transition entre tradition hypertextuelle et nouveauté du design monopage, cette période offre l'opportunité d'étudier la relation entre l'émergence d'un dispositif techno-sémiotique et les systèmes de valeurs mobilisés jusque-là par les concepteurs et les lecteurs de sites web. Nous chercherons notamment à démontrer 
comment l'abandon de l'hypertexte, au profit du design monopage, reflète un souci dénoncé par Emmanuël Souchier (1996), Yves Jeanneret et Jean Davallon (2004) de concevoir l'hypertexte non plus comme le monde interconnecté décrit par les ingénieurs, mais comme une expérience de lecture/écriture singulière. Nous verrons ainsi comment les reproches adressés à l'hypertexte concernent essentiellement la qualité de cette expérience. Le passage de l'hypertexte au design monopage trahit également une seconde forme de décalage, distinguant un discours scientifique, quelquefois trop distancié, d'un discours pratique adhérant davantage à l'objet de la description bien que dans l'incapacité de fournir des modèles théoriques généralisables. De fait, nous souhaitons décrire une sorte d'entre-discours (scientifiquepratique), mêlant la disponibilité des modèles théoriques à la variabilité del'objet. Dans cette optique, nous nous focaliserons sur les discours dits épisémiotiques des auteurs/lecteurs de sites web en analysant les paroles des webdesigners. Ces dernières élaborent des théories locales de l'hypertexte et du design monopage dont la pertinence peut conduire à la déstabilisation des conceptions théoriques dominantes.

Nous organiserons notre propos en trois parties : la première consistera en une revue des questions concernant l'objet du webdesign et la perspective épisémiotique dans le cadre d'une sociosémiotique des médias. La seconde s'intéressera aux promesses de l'hypertexte émises dans les années 90, ainsi qu'aux critiques et annonces d'abandon formulées plus récemment. Enfin, la troisième analysera les nouveaux espoirs fondés par la communauté des webdesigners sur les sites monopages. Pour étudier ce webdesign, c'est-à-dire la représentation de l'information et le parcours que proposent les interfaces-écrans (PIGNIER; DROUILLAT, 2008, p. 28), notre 
corpus sélectionnera et exposera deux lieux de cristallisation des oppositions: l'expérience narrative (storytelling) et l'expérience de navigation par défilement (scrolling).

\section{Une perspective sociosémiotique sur les discours épisémiotiques}

Pour commencer, notre approche privilégie une sociosémiotique des médias cherchant à «inscrire dans le social l'analyse des productions médiatiques» (ABLADI; DUCARD, 2009, p. 145). Dans ce cadre, nous visons la description de formes reconnaissables par les usagers, en circulation dans la mémoire sociale et conditionnant la signification des sites web. Plus précisément, nous nous attarderons sur la coexistence polémique des deux prédilections sémiotiques (SOUCHIER; JEANNERET; MAREC, 2003) que constituent l'hypertexte et le design monopage. Afin d'étudier cette confrontation entre deux modes de reconnaissance et de jugement du texte numérique, nous mobiliserons la notion de discours épisémiotiques, toujours selon une approche sociosémiotique. En nous référant aux travaux de Sémir Badir (BADIR, 2014, cap. 7), l'épisémiotique mobiliserait les représentations des sujets parlants et s'opposerait en ce sens aux descriptions scientifiques dites métasémiotiques. Les discours épisémiotiques, notamment produits par ce qu'Antoine Culioli nomme «la glose» (CULIOLI; NORMAND, 2005), seraient une parole schématisante, plus dynamique et plus générale que celle produite par le discours métasémiotique. Cette prise en compte étendue de l'activité épilinguistique engendrerait une ouverture des possibles vis-à-vis de la théorie, débusquant des invariances à même 
l'objet, à un «[...] niveau sous-jacent à l'objet ordinaire étudié par les linguistes» (BADIR, 2014, cap. 7).

Si cette glose sur l'objet nous paraît difficilement accessible pour la présente étude, nous en mobiliserons néanmoins une version sociosémiotique consistant, en référence aux remarques de Badir (2014, cap. 7), à répartir les textes de notre corpus en diverses catégories de représentations. Nous restreindrons ainsi la notion d'épilinguistique à un «déterminant de représentations conscientes que le sujet peut produire sur la langue et le langage» (BADIR, 2014, cap. 7). Par ce choix, nous cherchons à valoriser l'écart entre la description scientifique et pratique des technologies sémiotiques, en nous intéressant à la critique de cet hypertexte et à la louange adressée au design monopage. Nous nous éloignerons donc, le temps de cet article, de la conception propre à Sémir Badir pour adopter davantage celle de Jean-Marie Klinkenberg, désignée par perspective émique:

La perspective émique est donc celle qui prend en considération les fonctions que les usagers attribuent euxmêmes à leurs pratiques sémiotiques. Elle se fonde sur l'étude des représentations qu'ils s'en font. Cette étude, on peut la mener à partir de ce que nous appelons ci-après les discours épisémiotiques (KLINKENBERG, 1996, p. 128).

On appellera discours épisémiotique les jugements que les usagers formulent à l'endroit de leurs pratiques sémiotiques ou des pratiques de ceux qu'ils observent [...] et qui permettent au sociosémioticien d'étudier le vaste domaine des codes de représentation (KLINKENBERG, 1996, p. 280).

Notre approche s'inspire également des pratiques linguistiques désignées comme «linguistique populaire» ou 
«folk linguistique» par Marie-Anne Paveau (PAVEAU, 2008) et décrites comme des élaborations métalinguistiques profanes, spontanées ou naïves. Celles que nous explorerons concernent les commentaires postés dans les blogs et les forums de discussion sur internet par des professionnels du webdesign. Nous espérons que cette perspective, que nous pourrions à notre tour qualifier de folk sémiotique (ou sémiotique populaire), aura le mérite d'enrichir les schématisations théoriques actuelles par l'adoption d'une position dite «intégrationniste» ou «anti-éliminative» des données folk à la science (PAVEAU, 2008). Ces données, aux allures de glose sur l'objet, devraient nous rapprocher davantage de l'activité épisémiotique en nous offrant un accès possible aux expériences perceptives et cognitives des sujets énonçants. Autrement dit, elles autorisent l'étude d'une dimension expérientielle et culturelle du langage, celle des «usages de la langue par des sujets sociaux et cognitifs» (PAVEAU, 2008).

\section{Des métaphores d'apparences aux métaphores d'actions}

L'apparition de l'hypertexte dans les années 90 ne s'apparente justement pas, dans les descriptions qui en sont données, à une apparition vécue de l'intérieur par l'usager d'un internet encore balbutiant. Comme le décrit Gérard Verroust dans l'entrée de l'encyclopédie Universalis, l'hypertexte se présente comme des nœuds connectés par des liens qui mettent indéfiniment en relation des pages, des textes, des parties de texte, des mots, des images et des documents sonores, voire d'autres hypertextes (VERROUST, [s.d.]). Cette place nouvellement donnée à l'interconnexion d'éléments 
signifiants n'étant pas directement expérimentable par l'usager, les descriptions des années 90 ont eu massivement recours à la médiation de métaphores chargées de rendre compte de cette nouvelle structure hypertextuelle et de la rupture qu'elle constituait avec la tradition du texte. Les métaphores de l'étoile et de la corde à nœuds, proposées par Pierre Lévy, en sont un exemple:

Les items d'information ne sont pas reliés linéairement, comme sur une corde à nœuds, mais chacun d'eux, ou la plupart, étendent leurs liens en étoile, sur un mode réticulaire (LÉVY, 1991).

Si la notion de texte semble figurer un certain rétrécissement du cheminement (celui d'une ligne qui réduit les possibles du parcours), l'hypertexte au contraire se développe librement dans les trois dimensions de l'espace. Comme le dit Claude Bursztejn à propos du principe de topologie de l'hypertexte, «le réseau n'est pas dans l'espace, il est l'espace» (BURSZTEJN, 2004). Et la métaphore se poursuit par une sorte d'océanification de l'espace web dans lequel l'usager peut désormais naviguer ou surfer. Autant de métaphores d'actions associant l'espace web à une sorte d'immensité navigable, mais également prédéfinissant notre rapport renouvelé à l'information. En effet, comme le souligne si bien Claude Bursztejn, l'interaction à l'hypertexte peut être assimilée à la performance d'un surfeur qui se «déplace rapidement dans le creux des vagues, tout en cherchant à se maintenir d'une surface qu'il semble survoler» (BURSZTEJN, 2004). En creux, cette métaphore paraît dessiner une visée déontique, celle d'imposer une nouvelle relation aux objets culturels fondée sur la vitesse, l'immédiateté, mais également une certaine superficialité. 


\section{L'énonciateur: collectivité impersonnelle et hybridations}

Concernant l'énonciation hypertextuelle, nous pouvons filer la métaphore enévoquantles promesses de quitterles rives étroites d'un texte personnalisé par un ou plusieurs auteurs identifiables et de rejoindre le grand large informationnel d'hypertextes anonymes. L'énonciation collective s'en trouve ainsi modifiée dans le sens où la construction n'obéit plus à la succession des auteurs mais à une lente agrégation/fusion des individualités dans une collectivité désormais impersonnelle.

Ce n'est plus «chacun son tour » ou "l'un après l'autre» mais une sorte de lente écriture collective, désynchronisée, dédramatisée, éclatée, comme croissant d'elle-même suivant une multitude de lignes parallèles, et pourtant toujours disponible, ordonnée, objectivée sur l'écran (LÉVY, 1991).

Notons par ailleurs que ces individualités humaines s'agrègent et fusionnent avec des énonciateurs non-humains comme en témoigne Jean Clément:

La théorie hypertextuelle distingue entre les liens «calculés» (par la machine) et les liens "édités» (par un auteur). On l'aura compris: ce sont ces derniers qui nous intéressent ici. L'hypertextualisation automatique ne relève pas d'une écriture, les liens qu'elle produit n'étant que le résultat d'un calcul de la machine (CLÉMENT, 2007).

Si le calcul n'est pas considéré comme de l'écriture pour la théorie hypertextuelle, on peut toutefois se questionner dès lors qu'un usager expérimente la pageécran. Saura-t-il réellement faire la différence entre les deux 
types d'énonciateurs ? Dans le cas contraire, l'énonciation hypertextuelle deviendrait alors une co-opération à part entière, une action située dans laquelle la machine et l'humain se partageraient les rôles. Nous pouvons alors acter d'une certaine numérisation de l'auteur quand une partie du discours relève d'une énonciation machinique. Inversement, l'hypertexte promettait également une certaine humanisation de la technologie puisque, dès ses débuts, les chercheurs ont défendu un certain mimétismepostuléentrelefonctionnement cognitif des structures cérébrales et celui associatif des liens hypertextuels. Commel'affirmaient par exemple Patricia Baird et Mark Percival en 1989, «l'information peut être structurée dans l'hypertexte de la même manière que dans la tête de son auteur» (BAIRD; PERCIVAL, 1989, notre traduction) ${ }^{1}$. Pour Pierre Lévy, l'hypertexte se présenterait même comme une version extériorisée ou réifiée du processus de signification:

[...] En quoi consiste l'acte de donner du sens ? L'opération élémentaire de l'activité interprétative est l'association; donner du sens à un texte quelconque, revient à le relier, le connecter à d'autres textes, et donc à construire un hypertexte (LÉVY, 1991).

Cependant, Claude Bursztejn nuance ce mimétisme de la machine avec le mode associatif de la pensée humaine en dénonçant une certaine adaptation de l'humain à la machine qui privilégierait un recours outillé à l'associatif:

[...] si l'usage de plus en plus généralisé de l'hypertexte, tendant à privilégier un mode de pensée associatif sur le discours logique et sur la narration, n’a pas pour conséquence

1 "Information can be put into the hypertext in the same structure as it is in the author's head". 
une modification du fonctionnement cognitif, peut-être faut-il y voir une adaptation aux systèmes électroniques et informatiques qui, plus que des outils, deviendraient des prothèses quasi indispensables (BURSZTEJN, 2004).

Quelle que soit la description, cette co-énonciation partagée entre l'homme et la machine fait de l'hypertexte l'instigateur d'une figure de l'auteur en cyborg, d'un humain dont l'écriture serait sous la dépendance d'une prothèse technologique.

\section{L'hypertexte: machine à penser et expérience sensible}

Progressivement, nous remarquons à quel point la technologiehypertextuellese dégage de sa matérialité(systèmes électroniques ou structures cérébrales) pour rendre compte d'idéalités. Par exemple, le caractère social de l'interprétation emprunte les traits d'un «filet sémiotique» et «du coup, se trouve donné à voir et comme matérialisé la constitution du sens commun: l'élaboration collective d'un hypertexte» (LÉVY, 1991). Depuis le début de la description, nous relevons un certain déplacement de l'intérêt associé à l'émergence de l'hypertexte: l'objet ou la cible de la communication n'est plus le message, ni le couple classique émetteur/récepteur, mais le contexte sur lequel les acteurs de la communication agissent par des actions perpétuelles d'association et de dissociation qui déforment et remodèlent les univers de sens disposés (ou mis à disposition) par l'hypertexte. Ce déplacement d'intérêt s'accompagne également d'un déplacement du point de vue par l'adoption d'une vision externe. En effet, le constat d'émergence de l'hypertexte y est dépeint comme 
vue de l'extérieur, d'une position lointaine qui ne permet pas l'implication corporelle d'un quelconque émetteur ou récepteur humain. Cet éloignement conduit à une certaine abstraction de l'hypertexte, ou du moins à la privation de ses propriétés sensibles. Pour Pierre Lévy (1991), l'hypertexte est un réseau de significations toujours en construction et renégociation, une entité que nous qualifierons de polysémiotique, car associant textes, images, sons et vidéos. Ailleurs, il apparaît comme un jeu d'emboîtements récursifs de réseaux et de sous-réseaux, métaphore que l'on imagine prenant source dans les théories fractales en vogue à la même époque (je pense notamment à l'art fractal chez Susan Condé ou aux écrits du philosophe Edgar Morin sur la complexité).

Pour contrecarrer cette vision externe dominante, des chercheurs en sciences de l'information et de la communication ont cherché à reprendre ces descriptions du point de vue d'une vision interne: celui de l'expérience. Ainsi, une histoire de l'écriture informatique et plus précisément de l'hypertexte reste à réécrire si l'on adopte le regard des usagers, des auteurs ou des lecteurs qui, telle une fenêtre, donnent sur un écran. Cette perspective de l'hypertexte comme «écrit d'écran» est l'une des propositions d'Emmanuël Souchier (1996) qui correspond selon nous le plus à ce changement de point de vue sur la technologie sémiotique. Le chercheur y conçoit cette vision interne comme une réhabilitation possible de l'histoire du texte «à travers l'espace de l'écran» (SOUCHIER, 1996). Notons qu'un rapprochement entre l'expérience des écrits d'écran et notre dimension épisémiotique est d'ailleurs possible par le recours à l'infra-ordinaire (JEANNERET; DAVALLON, 2004; SOUCHIER, 1998). Cette notion vise la description des mécanismes cachés derrière les évidences de l'expérience quotidienne ce qui, dans le cas de l'hypertexte, ne manque pas 
d'engendrer désillusions et commentaires critiques.

Aussi, les constats les plus récents font état d'un rendezvous manqué avec l'hypertexte. Tout d'abord dans le domaine des études scientifiques, nous sommes passés à côté des usages et des usagers de l'hypertexte:

Les commentaires [...] tendent à évacuer l'acte d'écriture et de lecture, l'acte de mise en forme, l'acte de manipulation d'un dispositif, celui de configurer des médiations sociales et d'inscrire l'initiative dans un contexte pratique (JEANNERET; DAVALLON, 2004).

Dès lors que les liens deviennent des signes passeurs offerts aux regards, la description prend l'apparence d'une «métasémiotique implicite de l'hyperlien» (JEANNERET; DAVALLON, 2004) dont on ne peut nier une nouvelle fois la proximité avec la dimension épisémiotique présentée en début d'article. Pareillement, Alexandra Saemmer évoque ce potentiel sémantique insuffisamment exploité «entre texte géniteur et textes reliés, ou encore entre geste interactif et textes associés» (SAEMMER, 2011). Autant de rendez-vous manqués avec ce corps de l'énonciateur, inscrit dans un espace-temps hypertextuel en voie d'abandon. A ce propos, un regret paraît transparaître chez Caroline Angé, lorsqu'elle souligne que l'hypertexte «n'est plus à la mode en dépit des interrogations qu'il soulève» (ANGÉ, 2011). Enfin, le rendez-vous manqué concerne également la création littéraire hypertextuelle, dans les discours pratiques d'auteurs. Citons par exemple Dylan Kinette qui, s'interrogeant sur la mort annoncée de l'hypertexte, démontre paradoxalement que «le futur du livre n'est jamais arrivé» (KINETT, 2012, notre traduction) ${ }^{2}$.

2 "[...] the book's future never happened" 


\section{Futur démodé, écriture fragmentée et dépassement du modèle de l'imprimerie}

Pour aller plus loin, nous pourrions rechercher quelques raisons liées à cet abandon de l'hypertexte. Il semblerait que l'imaginaire développé par l'hypertexte n'ait pas présenté plus de résistance à la pratique technologique et sociale que ce que l'on nomme généralement un effet de mode. Ainsi, le caractère démodé de l'hypertexte est la première récurrence notable dans notre corpus. Selon Luc Dall-Armellina, «le terme d'hypertexte peut aujourd'hui passer pour déjà ancien, si ce n'est désuet» (DALL'ARMELLINA, 2009). Pour cette raison, l'hypertexte ne possèderait pas le caractère définitif annoncé dans les années 90, il ne représente au final qu'une expression de cette époque parmi d'autres. Dans le même sens, Dylan Kinett évoque clairement ce trouble à la lecture d'un futur démodé dans les poèmes hypertextuels rédigés dans les années 90 (KINETT, 2012).

Par ailleurs, les commentaires des webdesigners rendent compte de l'obstacle que peut engendrer l'hypertexte entre son usager et l'information recherchée. Cette épaisseur apparaît sous la forme d'une liste d'actions préalables (assimilable à un programme d'usage) que l'hypertexte est accusé d'allonger. Ainsi, l'usager devra trouver le lien, le cibler, le cliquer et attendre que la page soit chargée (ANTHONY, 2012); il devra également être capable de parcourir plusieurs pages (BURNS, 2013) pour trouver l'information recherchée. Le dispositif hypertextuel transforme alors la recherche d'information en une quête semée d'embûches, d'actions qui peuvent distraire l'usager au point de lui faire oublier le pourquoi de sa venue (PIVOT, 2015). Le flux continu de la lecture se trouve alors brisé par la transition entre page (DREW, 2015) et le contenu 
lui-même à tel point morcelé que Jean Clément n'hésite pas à inscrire l'hypertexte dans la lignée des textes fragmentaires (CLÉMENT, 2007).

Enfin, pour la plupart des webdesigners (BURNS, 2013; B WELLER, 2013; GALLI, 2012; JACOBS, 2014; LAVANDIER, 2013), l'hypertexte n'a jamais réellement représenté une révolution dans l'écriture numérique, du fait que sa conception est toujours restée soumise au modèle du livre et de l'imprimerie. Il s'agira alors de faire peau neuve en libérant les sites web des conventions dépassées de l'imprimerie et d'éléments archaïques tels que la page (B WELLER, 2013).

\section{Préfigurations post-hypertextuelles: chronologie et agglomération de l'information}

De nouvelles conceptions, de nouveaux modes d'écritures numériques commencent à voir le jour en réaction à ces modèles. Selon Florian Harmand, la métaphore du livre se trouve bousculée par l'arrivée de nouveaux modèles tels que les flux chronologiques des blogs et réseaux sociaux (HARMAND, 2013). L'hypertexte est donc poussé vers la sortie par la concurrence d'autres modèles plus récents et massivement utilisés par les usagers du net. Le premier de ces modèles est celui de la présentation chronologique de flux d'informations (ou fils). Facebook ou Twitter ont par exemple développé chez l'usager des comportements spécifiques de recherche d'information. Le second modèle concurrent est celui des cartes (card design), autrefois utilisé dans la conception informatique et consistant à présenter le contenu informationnel sous la forme de blocs de fonctionnalité et de sens. Cette agglomération concise de l'information s'inspire, 
selon Florian Harmand, du développement récent des applications web sur les smartphones dont la présentation est similaire. De cette manière, la concurrence du fil chronologique et de l'agglomération de l'information prépare le terrain, préfigure le design monopage et rend prévisible l'engouement qui en accompagne l'émergence actuelle. En rupture avec une certaine idée et expérience de l'hypertexte, on recherchera donc des solutions pour obtenir une expérience non-décousue (non-fragmentaire), dépourvue de toute chasse aux liens ou de clics aux effets incertains (nonassociatif) (B WELLER, 2013), on visera le rassemblement des informations sous forme de cartes, de blocs de données agencées pour diminuer le nombre de pages (non-réticulaire) (HARMAND, 2013).

\section{La (nouvelle) révolution du monopage}

Des noms seront rapidement trouvés pour baptiser ces nouveaux sites internet, tous porteurs d'une certaine hostilité envers le principal représentant de l'ancien modèle: la page. Les plus radicaux emploieront le terme de «pageless design», annonçant de la sorte sa disparition totale. Les plus accommodants parleront plutôt de page unique en utilisant indifféremment «single-page design» ou «one page design». Notons que la seule traduction française retenue à ce jour a choisi cette seconde option en retenant le terme «monopage». Conformément aux modèles préfigurés par la rupture avec l'hypertexte, nous retrouvons dans les sites monopages la notion d'agglomération évoquée précédemment. Par exemple, Emily Weeks considère le monopage comme un «encapsulement» des données d'un site entier dans une 
seule page «fluide» (WEEKS, 2013, notre traduction) ${ }^{3}$. Cette vision idéale de la centralisation d'un site sur une seule page est cependant nuancée, voire écornée, par les descriptions plus laconiques de certains webdesigners. Ainsi pour Simon Gombaud (GOMBAUD, 2014) ce type de site internet caractérise le plus souvent une longue page divisée en sections distinctes, chaque section correspondant à une thématique précise et disposant de ses propres objectifs : informer, séduire, convaincre ou encore pousser à l'action. La réticularité se produit donc de manière interne, dans une longue page désormais fragmentée de l'intérieur en sections de thématiques différentes. Cette fragmentation interne peut éventuellement se trouver renforcée par des signes plastiques telles que les couleurs comme le préconise Frederick Corbel (CORBEL, 2015). La navigation (browsing) dans l'espace de la longue page n'est plus externe comme dans l'hypertexte mais interne; on parle alors d'«inner-browsing» (GALLI, 2012). Pour cette raison, les sites monopages affichent une limite fondamentale, celle de la longueur de la page qui, reprenant les métaphores de Pierre Lévy, nous mettrait face à une trop longue corde à nœuds. Aussi, nous pouvons observer comment les discours des praticiens issus de cette mouvance vont chercher à justifier ce rallongement de la page.

\section{Efficacité des phrases courtes et habitudes de lecteurs jusqu'au-boutistes}

Puisqu'il n'y a qu'une seule page, de nombreux concepteurs considèrent ce dispositif d'écriture comme 3 "Pageless design, in short, is a single webpage that encapsulates an entire site's data into
one fluid page" 
une opportunité pour optimiser la qualité de ses contenus (TECHARK SOLUTIONS, 2015). Les informations étant condensées, le texte doit simplement être plus clair et plus concis selon Tabitha Burns (2013). Frederick Corbel préconise ainsi de limiter la longueur des textes en privilégiant les formules courtes etefficaces (CORBEL,2015). Lesitemonopage peut également devenir une option parmi d'autres et n'être sélectionné que dans les situations appropriées comme les contenus simples assimilables en quelques blocs (MARSH, 2012). Cependant, il est intéressant de remarquer comment cette écriture concise n'est pas uniquement le fruit de choix technologiques mais également celui de modèles sociaux qui contraignent les productions. Simon Gombaud évoque par exemple l'adage «parlez moins, dites plus» (GOMBAUD, 2014, notre traduction) ${ }^{4}$ auquel le dispositif technique est censé répondre. Il souligne plus loin comment cette volonté d'aller à l'essentiel s'inscrit dans un climat général de simplification des discours dans le but d'aérer les contenus (GOMBAUD, 2014). Un autre commentaire semble faire écho sur le blog de Frédéric Cavazza (2010), considérant les sites monopages comme un remède au phénomène de société de gavage informationnel des usagers nommé «infobésité».

Certains webdesigners justifient clairement le design monopage par les habitudes comportementales des usagers du web. Pour certains, la structure hypertextuelle a même été créée à partir d'une croyance erronée en des utilisateurs qui ne feraient pas défiler jusqu'en bas et ne traiteraient que la première partie de la page web (ANNA, 2013). Aujourd'hui, les choses ont changé puisque les usagers font défiler (scroll) la page jusqu'à la fin grâce aux comportements

4 "Talk less, say more". 
développés en fréquentant les réseaux sociaux (ANNA, 2013; ANTHONY, 2012; CORBEL, 2015). Quelque chose de tensif semble également se dessiner, car la quantité d'information semble reculer pour donner lieu à une intensification du contenu (JACOBS, 2014), une accentuation qui présage d'une mobilisation accrue de la sphère émotionnelle.

\section{De l'information à la narration: du clic au scroll}

Si le design monopage implique un changement radical des modes de pensées et de la manière de concevoir les sites web (ARRANZ SANTAMARIA, 2014), c'est bien dans l'émergence d'une narrativité toute nouvelle dans le monde du webdesign. D'un point de vue formel, nous voyons comment la disparition des menus, des arborescences et de la navigation par liens configure un discours linéaire propice au développement du narratif. En effet, la solution est toute trouvée pour la majorité des webdesigners: «on informe plus seulement, on raconte une histoire avec un déroulé et de belles illustrations» (LAVANDIER, 2013). Pour Nathan B. Weller, les récits représentent alors le moyen le plus simple pour délivrer des messages destinés à inspirer, motiver, susciter des actions et surtout faire progresser l'art de la narration (B WELLER, 2013). Cette narration vise à donner du relief aux transitions de la corde à nœuds du site monopage pour susciter la curiosité chez un internaute qui tentera de découvrir le cheminement du site pour y découvrir le contenu (CHOLET, 2012). L'expérience narrative transforme alors l'accès à un site informationnel de type catalogue, en une immersion narrative «dans les premières lignes d'une 
grande histoire» (B WELLER, 2013, notre traduction)5. Pour Chuck Longanecker, l'abandon de l'hypertexte est celui des disjonctions liées à l'interaction par sauts de pages. Par cette disparition, les webdesigners peuvent créer des histoires qui s'écoulent de manière continue au travers du site monopage (LONGANECKER, 2013).

Nous voyons alors comment le genre informatif ou narratif apparaît dépendant d'un mode d'interaction : le clic et le scroll. En effet, ce storytelling n'est pas seulement lié à un engouement momentané pour les histoires racontées, mais il semble essentiellement induit par un ensemble de facteurs environnementaux dont la structure des interactions numériques fait partie (HARMAND, 2013). Si l'hypertexte représentait le règne du clic de liens en liens, le coulant monopage instaure celui du défilement (scroll). Le verbe «scroller» utilisé en français dans le domaine du webdesign est un emprunt à l'anglais «to scroll» qui signifie faire défiler verticalement ou horizontalement le contenu d'un écran à l'aide de la molette de la souris, d'un écran ou d'un pavé tactile (CHOLET, 2012). Cette forme d'interaction paraît particulièrement adaptée à la narration puisqu'elle permet de parcourir le récit par une linéarité du geste. Cette compatibilité remarquable entre la forme d'écriture/lecture et la forme d'interaction exploratoire est telle qu'elle produit un effet de naturalisation en rendant l'interface intuitive (B WELLER, 2013). Du côté des usagers, ce changement d'interaction ne se présente pas comme un véritable changement culturel (ARRANZ SANTAMARIA, 2014), mais il s'inscrit depuis peu dans les habitudes, prenant le statut de seconde nature (ANNA, 2013). A cela s'ajoute la notion de

5 "You are immediately immersed in the opening lines of a great story". 
plaisir du défilement, souligné par Katie Fishburne lorsqu'elle considère que «[...] ces derniers temps, les gens scrollent, ils veulent scroller, alors laissons les scroller» (FISHBURNE, 2015, notre traduction) ${ }^{6}$.

Cependant, ce plaisir et cet engouement pour le défilement (scrolling) peuvent faire l'objet de critiques. La première concerne ce nouveau web qui nous fait trop scroller et toujours scroller au travers de sites qualifiés de «longscrolling» (CORBEL, 2015). Si ce scroll semble naturel, il ne s'agirait pas non plus de proposer des scrolling sans fin sous peine de toucher le fond (LE CAM, 2015) ou encore de martyriser nos yeux et nos molettes (CHOLET, 2012). Nous voyons alors comment un système de valeurs s'installe, le défilement obéissant à une norme de quantité maintenant la production entre l'insuffisance de défilement reprochée aux structures hypertextuelles et l'excès du design monopage. Ce changement d'interaction présente également des répercussions sur le traitement cognitif des informations par les usagers. En effet, citant John Herman, Katie Fishburne explique comment le clic relève plutôt d'un choix de l'usager, comme le fait de «sauter » alors que scroller apparaît comme inévitable, comme «tomber» (FISHBURNE, 2015). Ce design basé sur le défilement conduirait ainsi l'usager à une certaine passivité qui retarderait le moment de la prise de décision. De ce fait, l'action de scroller peut être opposée à celle de cliquer sur le terrain de l'implication du lecteur. Dans l'espace hypertextuel, l'usager doit prendre consciemment des décisions à propos de la suite du parcours du site. Il détient un certain contrôle et choisit ses "propres aventures» (FISHBURNE, 2015) en décidant où il va et quelles informations

6 "[...] these days people do scroll, they want to scroll, just let them scroll". 
l'y conduiront. En ce sens, l'hypertexte dessine en creux une identité de l'usager qui doit savoir ce qu'il veut (FISHBURNE, 2015). Différemment, l'usager d'un site monopage doit se laisser guider dans la narration pour que les premières lignes puissent susciter curiosité et désirs. Dans cette situation, l'usager apparaît comme un sujet démodalisé en attente d'un vouloir savoir, à la recherche de son désir de connaissance dans les diverses suggestions du site monopage. Inversement, s'il possède une idée bien précise de ce qu'il cherche (un vouloir-savoir propre), le site monopage apparaît beaucoup moins efficace pour trouver des informations pointues (FISHBURNE, 2015).

\section{Considérations finales}

Cette étude de la transition sémiotique de l'hypertexte vers le design de site monopage nous renseigne sur l'évolution des propriétés techniques et sémiotiques de ces dispositifs numériques mais également sur celle des points de vue adoptés par les chercheurs dans leur démarche d'analyse. Nous avons isolé une première perspective - première dans la découverte mais également dans la chronologie des publications scientifiques - centrée sur la description de «mondes». La nouveauté de l'hypertexte y est alors présentée comme une inconnue à mettre en mots connus, par la médiation de métaphores. Ainsi nous avons relevé l'usage récurrent de métaphores d'apparences (corde à nœuds et étoile) et de métaphores d'actions (cheminer et surfer). Toujours dans la même perspective, nous avons vu comment l'énonciateur était également décrit comme une entité individuelle identifiable pour le texte et une entité collective 
impersonnelle pour l'hypertexte. Nous avons noté au passage que cette collectivité incluait des énonciations calculées imputables aux machines. Pour conclure sur cette vision externe de l'hypertexte, nous avons démontré comment les descriptions tendaient vers une certaine dématérialisation de la technologie pour donner lieu à des abstractions, des modèles de pensée tels que la pensée associative, réticulaire ou encore la récursivité empruntée au modèle fractal. Aussi, une telle description distanciée, identifiable tant chez les ingénieurs que chez les chercheurs, semblait autoriser toutes les possibilités combinatoires de l'imagination et la formulation des promesses les plus extravagantes. Ainsi l'hypertexte promettait de quitter le cheminement du texte pour rejoindre l'océan informationnel; la toute puissance de l'auteur se trouverait renversée par une énonciation collective et impersonnelle dépendante de la technologie, l'humain se machiniserait et la machine s'humaniserait par le mimétisme entretenu avec le fonctionnement cérébral. Toujours vu de loin, les actions hypertextuelles se présenteraient comme autant de jets de pierre dans l'eau, dont les cercles concentriques bousculeraient les univers de sens mis à disposition.

Progressivement, dans sa phase de maturation, la réflexion sur l'hypertexte semble se confronter davantage aux réalités de ses usages et usagers. Ce glissement vers une vision interne requiert l'implication corporelle de l'analyste qui s'engage non plus dans la perception de l'entité hypertextuelle, mais dans celle dela pratique, del'expérience hypertextuelle. Il ne s'agit plus d'évaluer l'impact de l'hypertexte pour le monde, mais pour soi. La réhabilitation du texte enclenchée par la notion d'écrit d'écran a permis de déplacer l'hypertexte de son apparence technique vers son apparaître textuel. Aussi, nous devons souligner ce curieux 
constat que malgré l'abandon annoncé de l'hypertexte, nous n'en serions en réalité qu'au début de notre rencontre avec lui. Les spécialistes de l'écriture hypertextuelle ne manquent pas de souligner les rendez-vous manqués, notamment avec le faire, le sentir, le croire et le savoir propres aux expériences hypertextuelles.

Ainsi, l'hypertexte s'accompagne paradoxalement d'une certaine désuétude de son apparence dans le monde numérique alors que la nouveauté de son apparaître pour soi reste d'actualité.

Dans notre corpus, nous avons remarqué comment les discours ordinaires des webdesigners reprenaient, dans leur critique de l'hypertexte, cette vision interne adoptée de manière tardive par les scientifiques. La justification de la transition semble essentiellement reposer sur des récits d'avaries expérientielles, de manière à souligner les aspects négatifs de l'hypertexte et préfigurer en creux les prochaines évolutions numériques. L'hypertexte devient alors un obstacle (un parcours d'actions), une fausse révolution (une soumission au modèle de l'imprimerie), voire une écriture fragmentaire (un éparpillement du multipage). Autant de contributions à l'élaboration d'un contre-modèle à rejeter dont la page sera le parfait représentant.

La vision interne des discours ordinaires permet également de repérer des contraintes sociales pesant sur les dispositifs énonciatifs, telles que le climat de simplification des discours dans lequel a émergé le design monopage ou encore la lutte contre l'excès informationnel (l'infobésité). Les webdesigners sont eux-mêmes bien conscients de ces contraintes sociales pesant sur les choix technologiques, ces jeux de tendances, de stratégies éditoriales qui permettent de s'émarger d'une position dominante tout en veillant à ne 
pas être trop vite rejoints par la concurrence. Citons Tabitha Burns à ce propos:

Aujourd'hui, les sites monopages paraissent surprenants, contemporains, innovants et nouveaux. Mais si ce type de site est réellement le futur du webdesign, cela signifie que vous devez tirer avantage de sa singularité immédiatement, avant que le monopage ne devienne la norme (BURNS, 2013, notre traduction $)^{7}$.

Enfin, nous voyons également comment les modèles interactionnels liés à l'hypertexte (le clic) et au design monopage (le scroll) configurent la relation de l'usager avec les contenus informationnels. L'hypertexte dépeint un usager engagé dans une quête cognitive dans laquelle il devra prendre des décisions quant à son cheminement (cliquer comme sauter). Différemment, le design monopage présente un usager en quête de désirs, à la recherche d'un plaisir de connaître, simplement suggéré par le déroulement d'une l'histoire mise en scène le long d'une page unique (défiler comme tomber). Aussi, nous pouvons observer le jeu de solidarités entre un profil d'énonciateur, un choix technologique et un genre énonciatif. L'usager déterminé chercherait son information en cliquant dans un hypertexte, tandis que l'usager nonchalant ferait simplement défiler le contenu d'un site monopage.

Pour terminer, nous pourrions revenir sur la notion d'épisémiotique. Parmi les avantages tirés de l’adoption d'une perspective émique, nous pourrions évoquer une certaine accélération de l'investigation en accédant plus directement à la dimension expérientielle de toute technologie émergente. La

7 "Currently, pageless sites look impressive, contemporary, innovative and fresh. But if these types of site really are the future of web design, it means you must take advantage of their uniqueness now, before pageless sites become the norm". 
posture épisémiotique nous autoriserait également l'accès à la variabilité des objets sémiotiques sous le contrôle de la théorie. Dans les descriptions, elle nous permettrait de nous dégager des métaphores, des élaborations de l'imagination pour mettre les modèles théoriques disponibles à l'épreuve des retours d'expérience. Enfin, la diversification opérée par le recueil des discours naïfs ou spontanés permettrait également de revivifier la théorie par la brèche ouverte vers un antérieur au langage. Ce que Paul Ricœur ([s.d.]) associe à l'antéprédicatif, le primitif, voire l'être sauvage, rejoint cette démarche de la glose d'Antoine Culioli, cherchant par tous les moyens à reconditionner les modèles du dire sur ceux de l'apparaître.

\section{Références}

ABLADI, D.; DUCARD, D. (EDS.). Vocabulaire des études sémiotiques et sémiologiques. Paris: Honoré Champion, 2009.

ANGÉ, C. Introduction. Les cahiers du numérique, v. 7, n. 3, 2011.

ANNA. Is scrolling the new click? 2013. Disponível em: <http://webantic.co.uk/user-experienceux-design-isscrolling-the-new-click/>. Acesso em: 12 dez. 2015.

ANTHONY. Why Scrolling is the New Click. 2012. Disponível em: <http://uxmovement.com/navigation/why-scrolling-isthe-new-click/>. Acesso em: 12 dez. 2015. 
ARRANZ SANTAMARIA, J. M. The Single Page Interface Manifesto. 2014. Disponível em: <http://itsnat.sourceforge. net/php/spim/spi_manifesto_en.php>. Acesso em: 12 dez. 2015.

BADIR, S. Epistémologie sémiotique. Paris: Honoré Champion, 2014.

BAIRD, P.; PERCIVAL, M. Glasgow On-Line: database development using Apple's HyperCard. In: MCALEESE, R. (Ed.). Hypertext: Theory into practice. Oxford: Intellect, 1989.

BURNS, T. The Future of Web Design is Pageless. 2013. Disponível em:

$<$ http://blog.kualo.com/pageless-web-design/>. Acesso em: 13 dez. 2015.

BURSZTEJN, C. La pensée hypertexte. Le Coq-héron, v. 176, n. 1, p. 130-136, 2004.

B WELLER, N. 8 Reasons Why Pageless Design is the Future of the Web. 2013. Disponível em: <http://www.dtelepathy. $\mathrm{com} / \mathrm{blog} /$ design/8-reasons-why-pageless-design-is-thefuture-of-the-web>. Acesso em: 13 dez. 2015

CAVAZZA, F. Vive les sites web à page unique! 2010. Disponível em: <http://www.simpleweb.fr/2010/10/14/ vive-les-sites-web-a-page-unique/>. Acesso em: 13 dez. 2015.

CHOLET, S. One page design: effet de mode ou réel intérêt pour les marques? 2012. Disponível em: <http://www. presse-citron.net/one-page-design-effet-de-mode-ou-reelinteret-pour-les-marques/>. Acesso em: 13 dez. 2015 
CLÉMENT, J. L'hypertexte, une technologie intellectuelle à l'ère de la complexité. In: BROSSAUD, C.; REBER, B. (Eds.). Humanités numériques 1: nouvelles technologies cognitives et épistémologie. Paris: Hermès Science Publications, 2007.

CORBEL, F. Sites one page, long scrolling: et les contenus défilent..., 2015. Disponível em: <http://www.gazelle-duweb.com/ergonomie/sites-one-page-long-scrolling-et-lescontenus-defilent/>. Acesso em: 13 dez. 2015

CULIOLI, A.; NORMAND, C. Onze rencontres: sur le langage et les langues. Paris: Ophrys, 2005.

DALL'ARMELLINA, L. Hypertexte et Hypermodernité. In: SALEH, I. et al. (Eds.). H2PTM Rétrospective et perspective 1989-2009. Paris: Hermès Science Publications, 2009.

DREW, T. Using Motion For User Experience On Apps And Websites. 2015. Disponível em: <http://www. smashingmagazine.com/2015/01/using-motion-for-ux-onapps-and-websites/>. Acesso em: 13 dez. 2015.

FISHBURNE, K. Scrolling is Inevitable: Weighing in on Scrolling vs. Clicking in Web Design. 2015. Disponível em: $<$ http://vibethink.com/scrolling-inevitable-weighingscrolling-vs-clicking-web-design/>. Acesso em: 13 dez. 2015

\section{GALLI,M.Inner-browsingextendingthebrowsernavigation} paradigm. 2012. Disponível em: <https://developer.mozilla. org/en-US/docs/Inner-browsing_extending_the_browser_ navigation_paradigm>. Acesso em: 13 dez. 2015. 
GOMBAUD, S. Les sites “one page”, tendance passagère ou véritable orientation du Web en 2014 ? 2014. Disponível em: <http://blog.useweb.fr/2014/04/08/simon-gombaudles-sites-one-page-tendance-passagere-ou-veritableorientation-du-web-en-2014-2/>. Acesso em: 13 dez. 2015.

HARMAND, F. Standards du web et innovation. Allier créativité et standardisation, 2013. Disponível em: <http:// www.florian-harmand.fr/wp-content/themes/hfolio/files/ Standardisation_et_innovation_v1.pdf>. Acesso em: $13 \mathrm{dez}$. 2015

JACOBS, S. Does single page website perform well? 2014. Disponível em: <http://www.kgntechnologies.com/singlepage-website-perform-well/>. Acesso em: 13 dez. 2015.

JEANNERET, Y.; DAVALLON, J. La fausse évidence du lien hypertexte. Communication \& langages, n. 140, p. 43-54, 2004.

KINETT, D. The Death of Hypertext? 2012. Disponível em: <http://nocategories.net/ephemera/the-death-ofhypertext/>. Acesso em: 13 dez. 2015.

KLINKENBERG, J.-M. Précis de sémiotique générale. Paris: De Boeck Université, 1996.

LAVANDIER, S. Responsive et pageless design: vers un changement de l'écriture web? 2013. Disponível em: <http:// www.ecrirepourleweb.com/webdesign-2014-vers-unbouleversement-des-codes-decriture/>. Acesso em: 13 dez. 2015. 
LE CAM, N. Site one-page - Monopage mais multicarte? 2015. Disponível em: <http://blog.lunaweb.fr/site-one-page/>. Acesso em: 13 dez. 2015.

LÉVY, P. L'hypertexte, instrument et métaphore de la communication. Réseaux, v. 9, n. 46, p. 59-68, 1991.

LONGANECKER, C. Stop Building Websites and Start Building Smart Sites. 2013. Disponível em: <http://www. dtelepathy.com/blog/news-events/impress-smart-site>. Acesso em: 13 dez. 2015

MARSH, J. What are the UX benefits of one-page websites? 2012. Disponível em: <http://www.quora.com/What-arethe-UX-benefits-of-one-page-websites $>$. Acesso em: $13 \mathrm{dez}$. 2015.

PAVEAU, M.-A. Les non-linguistes font-ils de la linguistique? Pratiques. Linguistique, littérature, didactique, n. 139-140, p. 93-109, 15 dez. 2008.

PIGNIER, N.; DROUILLAT, B. Le webdesign: sociale expérience des interfaces web. Paris: Hermes Science Publications, 2008.

PIVOT. Why one-page website design is in vogue. 2015. Disponível em: <http://www.pivotcomm.com/insights/onepage-website-design-vogue>. Acesso em: 13 dez. 2015

RICCEUR, P. ONTOLOGIE. Disponível em: <http://www. universalis.fr/encyclopedie/ontologie/>. Acesso em: $13 \mathrm{dez}$. 2015. 
SAEMMER, A. Hypertexte et irradiation iconique. Les cahiers du numérique, v. 7, n. 3-4, p. 47-69, 2011.

SOUCHIER, E. L'écrit d'écran, pratiques d'écriture \& informatique. Communication et langages, v. 107, n. 1, p. 105-119, 1996.

SOUCHIER, E. L'image du texte pour une théorie de l'énonciation éditoriale. Les cahiers de médiologie, v. 6, n. 2, p. 137-145, 1998.

SOUCHIER, E.; JEANNERET, Y.; MAREC, J. L. Lire, écrire, récrire: objets, signes et pratiques des médias informatisés. Paris: Bibliothèque Publique d'Information, 2003.

TECHARK SOLUTIONS. Advantages of Single Page Websites. 2015. Disponível em: <http://www.gotechark. com/advantages-single-page-websites/>. Acesso em: $13 \mathrm{dez}$. 2015

VERROUST, G. Hypertexte. Disponível em: <http://www. universalis-edu.com/encyclopedie/hypertexte/>. Acesso em: 14 dez. 2015.

WEEKS, E. The Future of Web: Pageless Design. 2013. Disponível em: <http://blogs.imediaconnection.com/ blog/2013/08/12/the-future-of-web-pageless-design/>. Acesso em: 14 dez. 2015. 
DE L'HYPERTEXTE AU DESIGN MONOPAGE: UNE TRANSITION SÉMIOTIQUE

Artigo recebido em setembro de 2015 e aprovado em dezembro de 2015.

Disponível em: http://seer.fclar.unesp.br/casa 